\title{
Getting the Best of Both Worlds? Developing Complementary Equation-Based and Agent-Based Models
}

\author{
Claudius Gräbner ${ }^{1}(\mathbb{D})$ Catherine S. E. Bale ${ }^{2} \cdot$ Bernardo Alves Furtado $^{3}$. \\ Brais Alvarez-Pereira ${ }^{4}$. James E. Gentile ${ }^{5}$ - Heath Henderson ${ }^{6}$. \\ Francesca Lipari ${ }^{7}$
}

Accepted: 6 October 2017 / Published online: 1 November 2017

(C) The Author(s) 2017. This article is an open access publication

\begin{abstract}
We argue that building agent-based and equation-based versions of the same theoretical model is a fruitful way of gaining insights into real-world phenomena. We use the epistemological concept of "models as isolations and surrogate systems" as the philosophical underpinning of this argument. In particular, we show that agent-based and equation-based approaches align well when used simultaneously and, contrary to some common misconceptions, should be considered complements rather than substitutes. We illustrate the usefulness of the approach by examining a model of the long-run relationship between economic development and inequality (i.e., the Kuznets hypothesis).
\end{abstract}

$凶$ Claudius Gräbner

claudius@claudius-graebner.com

1 Institute for the Comprehensive Analysis of the Economy (ICAE), Johannes Kepler University Linz, Aubrunnerweg 3a, 4040 Linz, Austria

2 School of Chemical and Process Engineering and the School of Earth and Environment, University of Leeds, Energy Building, Leeds LS2 9JT, UK

3 Institute for Applied Economic Research (Ipea-Brazil), BS Quadra 1, B1. J., Brasília, DF 70076-900, Brazil

4 Department of Economics, European University Institute, Villa La Fonte, Via delle Fontanelle 18, San Domenico di Fiesole (FI), 50014 Florence, Italy

5 Two Six Labs, LLC, 4350 N. Fairfax Dr., Suite 410, Arlington, VA 22203, USA

6 College of Business and Public Administration, Drake University in Des Moines, Aliber Hall, 2507 University Avenue, Des Moines, IA 50311, USA

7 The Philosophy, Politics and Economics Program, University of Pennsylvania, 375, Cohen Hall, 249 S 36th Street, Philadelphia, PA 19104, USA 
Keywords Agent-based models · Epistemology · Equation-based models · Economic methodology $\cdot$ Social simulation

\section{Introduction}

During the past three decades, agent-based models (ABMs) have become an increasingly prominent research method in economics. This raises important questions regarding their relationship with more traditional equation-based models (EBMs) in economics, and whether the two approaches can be reasonably demarcated from each other. Currently, much of the literature considers them to be mutually exclusive approaches to economic modeling (Van Dyke Parunak et al. 1998; Durlauf 2012). Here we will argue instead that the two approaches should be viewed as complements rather than substitutes.

The first thing to note is that a clear-cut distinction between the two approaches is not straightforward. There are at least two reasons for this: First, from a technical perspective, ABMs always consist of equations. Due to the Church-Turing thesis, every computer program could equally be expressed as a recursive function and many ABM include equations explicitly (Epstein 2006). Secondly, the criterion that ABMs are usually solved numerically is misleading. For example, dynamic stochastic general equilibrium (DSGE) models are often contrasted with ABMs in macroeconomics, yet almost all DSGE models involve numerical simulations.

Some argue that there are particular features such as agent heterogeneity, direct interaction, or bounded rationality that distinguish ABMs from EBMs (Epstein 2006). ${ }^{1}$ But given the progress made in traditional economic modeling to include these features into EBMs, these criteria do not seem to provide a sharp distinction either. Yet another demarcation line could be the use of the equilibrium condition usually employed in EBMs. ABMs often begin with assumptions on the behavior of economic agents and then derive the systemic dynamics via simulation. But these dynamics are frequently characterized by certain stable attractors that could be characterized as equilibria, and not all ABM lack a general equilibrium (Henderson and Isaac 2017; Johnson et al. 2017).

We argue that a strictly dichotomous distinction is not helpful. Rather, a useful and pragmatic way of distinguishing the two approaches refers to their epistemological foundation. For this reason, we rely on our interpretation of the epistemological concept of "models as isolations and surrogate systems" (MISS) as the philosophical underpinning of our argument (Mäki 2009a). This framework is useful for a number of reasons: (1) it shows how abstract economic models carry information about the system under investigation (SUI); (2) it helps highlighting the most fundamental differences between ABMs and EBMs; and (3) illustrates how the two approaches can be successfully combined and their mutual strengths exploited.

We show how a stylized EBM can serve as the starting point for a more refined $\mathrm{ABM}$ of a given theoretical model. In the end, both can be used to generate insights about the common SUI. We contend that this strategy helps to preserve the clarity

1 Also see the literature review provided in Sect. 3. 
and transparency of conventional EBMs, but permits a more extensive exploration of the system with ABMs. We advocate building models sequentially, with the ABM containing the (often very stylized) EBM as a special case (e.g. a case without heterogeneity among the agents or a trivial interaction network). For some configurations, both models should yield the same behavior. This can be used to test whether certain model features have been accurately reproduced and serves as a first verification of the ABM. ${ }^{2}$ Then, we can use the ABM to explore the model in more dimensions (e.g. by allowing for heterogeneity of agents or by activating additional mechanisms).

This additional step improves the explanatory power of the model through a couple of channels. ${ }^{3}$ First, one is able to judge the similarity of the model and the SUI across more aspects than in the purely equation-based form. The reason is that the algorithmic language of ABMs allows the consideration of aspects that are important in verbal models but cannot be accounted for in pure equation-based models. Secondly, ABMs usually provide more opportunities to calibrate and validate the model with empirical data, particularly on the micro- and meso-levels. ${ }^{4}$

The resulting increase in the complexity of the model is often warranted but bears the danger of causing confusion. Starting with a simple equation-based version and increasing the model's complexity stepwise helps to preserve its clarity. Also, the fundamental mechanisms of the model can usually still be communicated easily via precise equations. We further explore the different channels through which this procedure expands the explanatory content of the models in the next section. For now, let a simple example illustrate the argument.

Albin and Foley (1992) built an agent-based version of the Arrow-Debreu economy (Arrow and Debreu 1954), and showed how the absence of the Walrasian auctioneer leads to increasing inequality as a consequence of decentralized trading. The transformation of the EBM into an ABM thus allowed the authors to explore an additional dimension through stronger decomposition. In particular, it showed the impact of decentralized trading and the effect of different network structures underlying the trading relations of the agents. This was not only valuable in itself, but it also increased the explanatory power of the original EBM. That is, it became easier to interpret the $\mathrm{EBM}$ as it permitted the authors to examine model outcomes change if the degree of decentralization gets increased.

Based on a sound epistemological concept and illustrated by an empirical application, our paper contributes to the literature by providing additional arguments for the complementarity and mutual advantage of EBMs and ABMs. Section 2 introduces the MISS framework and contains a theoretical discussion of the epistemological underpinnings of our claim. Section 3 builds upon the meta-theoretical vocabulary

\footnotetext{
2 See Axtell et al. (1996) for a more extensive discussion of the meaning of "yielding the same result."

3 To be precise about how the explanatory content of a model is increased requires an explicit epistemological framework such as the MISS. Since we will not introduce this framework until the next section, in this paragraph we simply assert that the explanatory power of the model is increased through the measures discussed here.

4 There are several approaches of bringing ABMs to the data and all have their advantages and disadvantages. See Fagiolo et al. (2007) for an excellent discussion. But note that there are many useful ABMs (and EBMs) that are not validated with data but remain theoretical. Most of our arguments here apply to both empirical and theoretical models.
} 
introduced in Sect. 2 and summarizes the existing literature on the relationship between ABMs and EBMs. Section 4 illustrates our arguments with a detailed application. In light of this application, Sect. 5 provides additional discussion regarding the usefulness of our approach and Sect. 6 concludes.

\section{Epistemological Considerations}

When using models in the social sciences, one must provide an answer to the question of how one's model explains something about the real world, even if the model's assumptions are not descriptively accurate. This simple question has received considerable attention in the philosophy of science and many answers have turned out to be incorrect or inconsistent. Most famously, Friedman (1953) suggested to focus on the capacity to provide good predictions rather than the descriptive adequacy of model assumptions. This proposal has been heavily criticized, particularly in the social sciences (see Musgrave (1981) and further discussion below).

The way one answers this fundamental question has important consequences for the extension of EBMs to ABMs. There are different concepts within the social sciences and economics that are used to give formal models an epistemic meaning. These different concepts entail different "weights" for the strengths and weaknesses of ABMs and EBMs. For example, for someone adhering to Friedman's epistemology, the possibility of considering more realistic assumptions in ABMs would not necessarily be beneficial. We apply here a particularly appropriate and consistent epistemological framework called "models as isolation and surrogate systems" (MISS) (Mäki 2009a, b). The approach is similar to the concept of models as "credible worlds" (Sudgen 2000), and illustrates how the proposed integration of EBMs and ABMs increases our knowledge of the SUI.

In the MISS approach, models are considered to have two fundamental aspects. First, they represent the real world. As reality is too complex to be understood directly, we reduce its complexity by abstracting details, thus building a reduced picture of reality. These pictures are referred to as either surrogate models or substitute models (Mäki 2009a). A surrogate model results from an active and reasonable attempt to learn something about reality. A substitute model results if the act of representing reality was a failure, either because the researcher did not accomplish their goal (e.g. by choosing a wrong form of representation) or because they simply want to study the model for its own sake. ${ }^{5}$

The second aspect of a model is that of resemblance. If reality is observed at two successive points of time, some of its properties usually have changed. Yet, the underlying mechanisms of this change are often not directly perceivable. Therefore one studies the model as a tractable representation of reality. If one records the state of the model at two points of time, the state has changed due to the mechanisms built into the model. Such a study of the behavior of the model is called model exploration.

\footnotetext{
5 To judge whether the attempt to represent the real world via a model usually also requires ontological considerations. Only if we make a claim about what exactly reality is can we argue that a certain model has failed to represent it. Such considerations go beyond the scope of this paper.
} 


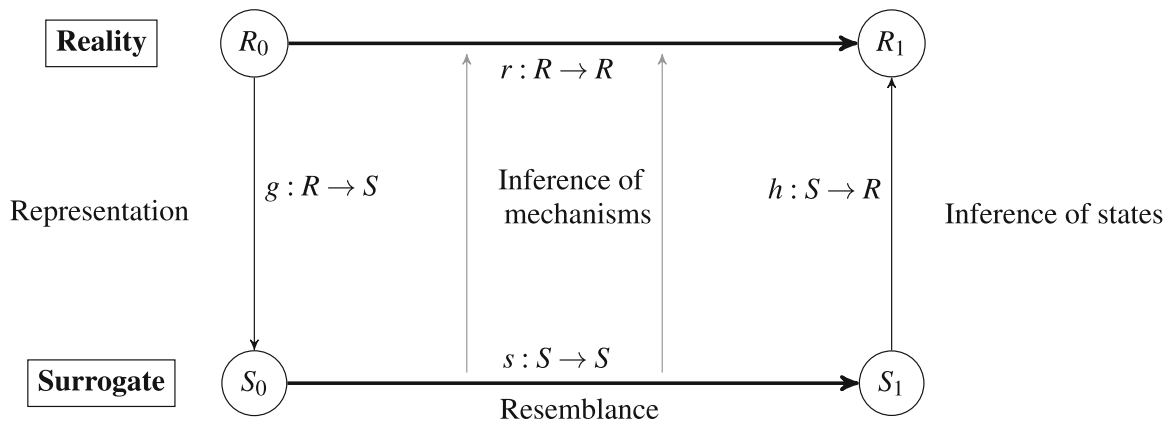

Fig. 1 The MISS concept interpreted as a mapping process (Gräbner 2017a)

The model exploration can take several forms depending on the model characteristics: If the model is purely verbal, the model exploration may be a thought experiment. For analytical models it may take the form of a mathematical proof, and for computational models it may take the form of a simulation. If we can learn something about the real world by the exploration of our model, the model resembles the real world and we have successfully increased our understanding of reality.

The act of "understanding reality" can be interpreted in at least two ways. In the first case, one is interested in how certain variables in the real world change over time. One then wishes to infer states of the real world. "How does GDP growth per capita evolve over time?" would be a typical question. The crucial inference would be from the variable GDP in the model to the variable GDP in the real world. In the second case, one is interested in the mechanisms operating in the real world. That is, one wishes to infer from the mechanisms built into the model to the mechanisms operating in reality. "Why does GDP evolve as it usually does?" or "What are the determinants of income inequality over time?" would be typical questions for this case.

Figure 1 illustrates the fundamental idea of the MISS approach. The modeler builds a surrogate $S_{t}$ of reality $R_{t}$ at time $t=0$, which is of lower complexity than reality itself. This process can be thought of as a mapping $g$ from reality to the model. The processes that drive the dynamics of the model can be thought of as a mapping $s$ and the processes that drive the dynamics of reality can be thought of as a mapping $r$. After the model has evolved from $S_{0}$ to $S_{1}$ at time $t=1$, one can then compare the resulting system $S_{1}$ with reality $R_{1}$ via the mapping $h$ (i.e., comparing the resulting "states"). Or, one may assess the plausibility of the mechanisms operating in the model compared with reality, which may or may not lead to a similarity between $R_{1}$ and $S_{1}$.

Figure 1 clarifies why a sole focus on predicting the right variables is not sufficient to guarantee a model's usefulness for understanding the mechanisms operating in the real world. One may either infer states correctly without having used adequate mechanisms in the model (a common drawback of purely instrumental models). Or, one may make false predictions despite having considered the right mechanisms. In Arthur's (1989) model of technology choice, for example, it is impossible to predict which technology will be the dominant one (or even if a single technology becomes dominant), but the behavior of the model is very well understood. 
The MISS framework thus illustrates that both dimensions, the representation and resemblance aspect of a model, are important. This makes it useful for our present purpose since it provides a straightforward way to view a phenomenon from an equation-based and an agent-based perspective. EBMs are usually very exact in the process of model exploration. This is because all mechanisms are expressed via clear equations and verification can take the form of a rigorous proof. ABMs, on the other hand, usually are not amendable to proofs and must be simulated. But a single simulation only represents one potential trajectory through the state space of the model and even more elaborate statistical analysis of the results does not offer the certainty of a mathematical proof.

On the other hand, the ability of EBMs to represent the real world is usually more restricted than that of ABMs. Due to tractability considerations, EBMs have fewer dimensions than ABMs. That is, EBMs require a greater reduction of complexity and include fewer variables than ABMs. This means that the ability of EBMs to represent reality is more restricted, and that they can be explored in fewer dimensions than ABMs. In particular, there are certain mechanisms that are very difficult to represent in an EBM. Examples include learning (Furtado and Sakowski 2014), true uncertainty (Pyka and Fagiolo 2007), or the endogenous formation of preferences (Hodgson and Knudsen 2004). ${ }^{6}$ This remains true even if, as noted above, important progress in conventional economic modeling has been made in incorporating some of these aspects into EBMs.

The greater flexibility of ABMs also means that models can be tested for the importance of implicit assumptions made during the model building process (Rahmandad and Sterman 2008). Consider the role of aggregation mechanisms as a practical example. In contrast to EBMs, ABMs are often used to explicitly study the aggregation mechanisms generating the macro observables of the system. EBMs often start with such macro observables and assume a certain aggregation mechanism as given (Van Dyke Parunak et al. 1998). By comparing the ABM (where the aggregation mechanism is explicit) with the EBM (where the aggregation mechanism is implicit), one may examine whether an explicit consideration of the aggregation mechanism is necessary. Again, the MISS framework makes clear what happens on the meta-theoretical level: the ABM helps in understanding whether the assumed complexity reduction function (function $g$ in Fig. 1-which, among other abstractions, contains the assumed aggregation mechanisms) of an EBM is appropriate and whether the EBM is an adequate surrogate of the SUI. As such, the ABM has increased the explanatory meaning of the associated EBM. ${ }^{7}$

\footnotetext{
6 Some also argue in favor of the relevance of considering the role of downward effects originating from social structures (e.g., Andersen et al. 2000) instead of reducing the analysis to the traditional methodological individualism. An interesting compromise could be a (epistemological and methodological) "systemist" approach to economics that allows for both upward and downward effects. Such an approach would certainly be compatible with ABM, but more difficult to realize via EBM. See Gräbner and Kapeller (2015) for a short introduction.

7 There is another reason for a joint use of ABMs and EBMs, even if one does not accept the distinction between modelling states and mechanisms. Gilboa et al. (2014) interpret models as analogies to which real cases can be compared. If social scientists want to make a statement about a real case, they can compare the real case with models, weighing the information contained in the models according to their perceived
} 
In summary, building agent-based versions of EBMs provides three primary benefits. First, ABMs allow exploration of the model in more dimensions, thereby improving inference and validation. Second, ABMs offer a constructive perspective on the problem under investigation and permit the consideration of mechanisms that are difficult to include in EBMs. Third, agent-based versions allow one to examine interaction effects between the isolated mechanisms of the EBM and other mechanisms operating in the real world. As EBMs are easier to verify and more precise for systems where such interaction effects can be overlooked, the strengths and weaknesses of EBMs and ABMs are complementary.

\section{Literature Review}

The economics literature comparing ABMs and EBMs is small. Furthermore, many arguments are not well grounded in the philosophy of science. This makes it difficult to relate the contributions from the various disciplines to each other. Fortunately, the meta-theoretical vocabulary introduced in the previous section proves useful in structuring the existing literature. We first discuss the literature that takes an explicitly meta-theoretical starting point and then move to applications where ABMs and EBMs are compared.

Epstein (2006) seeks to resolve a couple of misconceptions when it comes to the epistemology of ABMs. According to his concept of "generative sufficiency," models generate knowledge about social reality by reproducing observed dynamics as the outcome of the interaction of theoretical agents. While most ABMs align well with this notion, most EBMs do not. Thus, his approach represents a departure from conventional practice in economics, according to which a phenomenon is explained once it is derived as an equilibrium condition in a rational-choice model. Using the MISS vocabulary, Epstein requires that models not only produce outcome states in line with empirical evidence, but also that they resemble mechanisms that could reasonably operate in reality.

As an implication of the Church-Turing thesis, Epstein further clarifies that a corresponding recursive function exists for every algorithm and ABMs, thus, also consist of equations. He also shows that because many EBMs are in practice solved by simulation, the fact that a model is solved numerically does not qualify it as either an ABM or EBM. However, his own account of defining ABMs in terms of their features, such as heterogeneity and non-equilibrium dynamics, is problematic, as we have argued in Sect. 1.

Rust (1998) takes a different approach to demarcate ABMs from EBMs by arguing that in ABMs model dynamics are the result of the distributed computations of the agents, while in EBMs they are the result of the centralized computation of a set of

\footnotetext{
Footnote 7 continued

similarity with the real case. For example, when a solution for a two-player case holds for the multi-player case, the potential similarity of the theoretical model to the real world increases (Gilboa et al. 2014). The same is true when exploring an agent-based version of an EBM in a direction that has not been previously possible. Hence, building agent-based versions of EBMs increases the general amount of information contained in the models.
} 
equations. He also studies the computational complexity of economic computations and argues that only ABMs - as a particular form of parallel computation-allow for realistic representations of real economies that are still computationally tractable. No definition, however, is given as to what "realistic" means in the paper. Using the language of MISS, it means that ABMs can represent the essential properties of the SUI, and can resemble the mechanisms in an acceptable manner.

Rust (1998) paved the way for further studies looking at the computational complexity of economic processes. Axtell (2005) compares more precisely the computational complexity of centralized and decentralized exchange; the former associated with EBMs in the spirit of Arrow-Debreu and the latter associated with ABMs. Axtell analyzes the complexity of the computations required for decentralized and centralized exchange, arguing that the complexity of the latter is prohibitive. Decentralized trading of heterogeneous agents is considered a more realistic description. Importantly, he argues that by moving from the idea of a centralized to a decentralized market one vindicates several commonly held ideas about markets, such that they "do not disperse wealth, yield allocations that are determined solely by preferences and endowments and are not history-dependent" (Axtell 2005, p. F209). Note that his claim is on which mechanisms of exchange in models should be considered successful in resembling mechanisms in reality_not only on which results are plausible or not.

Apart from the few explicitly meta-theoretical considerations of the relationship between ABMs and EBMs, there are a couple studies that contrast the two approaches with a particular case study or explicitly build an agent-based (equation-based) version of an EBM (ABM). Maybe the most complete comparison of ABMs and EBMs comes from Rahmandad and Sterman (2008), who compare the two approaches in the context of contagious disease modeling. The authors contrast a mean-field differential equation model with an ABM for which using the same integration techniques and the same parameter values ensure comparability.

Rahmandad and Sterman (2008) find no significant differences between the two models for most of the key variables, and found the structure of the interactions to be unimportant if the EBM is fitted to the $\mathrm{ABM}^{8}{ }^{8}$ The effects of networks and agent heterogeneity are then absorbed by other parameters of the EBM. Following Axtell et al. (1996), the authors conclude that more models including both agent-based and equation-based aspects should be built. They also stress that ABMs are more advantageous over EBMs once data on the micro level is available. ${ }^{9}$ One implication of their argument is that ABMs have an advantage if one is interested in the particular mechanisms underlying the model outcomes, since the fact that the fitted EBM aligns effects of different network structures to other parameters does not increase our understanding of the underlying mechanisms.

Turning to applications in economics, ${ }^{10}$ one of the first direct comparisons comes from Albin and Foley (1992), whose work represents a classic example of taking a

\footnotetext{
8 See Cecconi et al. (2010) for similar conclusions.

9 See also Sukumar and Nutaro (2012) and Van Dyke Parunak et al. (1998).

10 We focus on applications within economics, but there are some particularly interesting case studies outside the social sciences. In epidemiology (e.g., Keeling 1999 or Halloran et al. 2002) and in ecology (e.g.,
} 
standard equilibrium model and turning it into an ABM (see Sect. 1). More recently, Gintis (2007) developed an agent-based version of the Walrasian general equilibrium model. In contrast to Albin and Foley (1992), this model features a replicator dynamics and private prices, but no explicit network structure. In doing so, Gintis substitutes the auction mechanism implicit in the equation-based version of the model by an explicit bargaining mechanism that can only be represented in an ABM. In contrast to analytically tractable EBMs, the resulting ABM features dynamic stability.

Oremland and Laubenbacher (2014) studied the relationship between EBMs and ABMs in the reverse direction. They describe a well-known ABM in the SugarScape model by using a system of difference equations that they use to find a parameter set that maximizes tax revenue within the ABM. In doing so, they support our claim of complementarity from an entirely different starting point. ${ }^{11}$ Another study that represents an application of the ideas presented here is Henderson and Isaac (2017), who extend an existing EBM on the organization of agrarian production to an ABM that more accurately represents modern agrarian economies. The authors build an ABM that matches the EBM in a simple specification, but can be extended to incorporate modern agricultural value chains. Their work is also distinctive in the sense that their ABM features both optimization by the agents and a general equilibrium. This example substantiates our criticism of defining ABMs in terms of non-equilibrium mechanics and non-optimizing agents.

Another contribution that deserves mentioning is the "docking" literature initiated by Axtell et al. (1996). The authors discuss when one model can be said to be a special case of a more general model. Interestingly, the authors make clear that it is not sufficient to look at the outcomes created by models, but rather the way the results have been generated must also be examined. Viewed from the perspective of the MISS, this means that models producing the same outcomes can do so with very different mechanisms. Two models producing the same outcome states via different mechanisms should certainly not be considered equivalent. ${ }^{12}$

In summary, by using the meta-theoretical language of the MISS we highlight a common claim of the few studies examining the relationship between EBMs and ABMs. ABMs have more potential for validation and are well suited for what we call mechanism-based explanations. EBMs are, however, easier to verify and represent pragmatic tools for predicting SUIs. There is indeed a growing number of studies in the literature suggesting a closer integration of the two approaches and in the following section we illustrate how this can be done.

\footnotetext{
Footnote 10 continued

Picard and Franc 2001), many papers take a similar approach to Rahmandad and Sterman (2008) by specifying an ABM, then fitting an EBM, and then comparing them to real data. Generally, these studies find that the results diverge more drastically the more important localized effects are for the dynamics of the system.

11 See Lafuerza et al. (2016) for a similar approach.

12 The MISS also links this with more fundamental ontological reasons, as the concept of a mechanism in the MISS relates to a mechanism in the philosophical concept of systemism. Since mechanisms are here considered an essential element of every system, two systems with a different set of mechanisms are necessarily distinct. It is therefore important to note that two models creating the same outcomes with different mechanisms are distinct. See Gräbner (2017b) for a more detailed discussion.
} 


\section{An Illustrative Example: The Kuznets Hypothesis}

In this section, we illustrate the usefulness of our approach by examining a model of the long-run relationship between economic development and inequality (i.e., the Kuznets hypothesis). We first discuss the underlying EBM and then explain how one might develop an ABM from the EBM. After this, we show the results from simulating the ABM and briefly discuss how the results enrich our understanding of observed historical experiences. The final subsection compares and contrasts the two model types, discussing the epistemological value-added of the approach as well as some practical difficulties.

\subsection{The Equation-Based Model}

Kuznets (1955) argued that income inequality dynamics follow an inverse U-shaped trajectory (called the Kuznets curve). That is, for a given country, inequality was said to increase during the early stages of development and then decrease after a certain level of development is reached. While Kuznets suggested that the phenomenon occurred naturally during industrialization because of dual economy dynamics, Acemoglu and Robinson (2002), henceforth AR, offered an alternative account that highlighted political factors. AR contended that "capitalist industrialization tends to increase inequality, but this inequality contains the seeds of its own destruction, because it induces a change in the political regime toward a more redistributive system" (p. 184). A major virtue of AR's model is that it not only explains the emergence of the Kuznets curve in some settings (e.g. Western Europe), but also explains important exceptions (e.g. East Asia). ${ }^{13}$

Here we offer a sketch of AR's model. The model is an infinite-horizon, nonoverlapping generations model with bequests. The model is characterized by a continuum of rational agents, each living for a single period and having one offspring. A fraction $\lambda$ of the agents are considered "poor" and the remaining are considered "rich." Let the subscript $p$ denote poor agents and the subscript $r$ denote rich agents. Agent type $i \in\{p, r\}$ possesses a unique asset $h_{i t} \geq 1$ at time $t$, which can be thought of as a composite of human capital, physical capital, and land. Agents use their assets to produce a unique consumption good $y$. A fraction of the revenue from the sale of the consumption good is then saved for educational bequests to their offspring, which is denoted by $e$. Importantly, if $e \leq 1$ a given agent will leave nothing to their offspring as the bequest is consumed.

The offspring's capital is governed by the following:

$$
h_{i, t+1}=\max \left\{1 ; Z e_{i, t+1}^{\beta}\right\}
$$

\footnotetext{
13 In the case of the "East Asian miracle," inequality remains low throughout the development process and the economy accumulates rapidly. Due to the fact that the gains from prosperity are relatively equally shared, social pressure and political reform are delayed.
} 
where $Z>1$ and $\beta<1$ to ensure that accumulation does not continue indefinitely. Eq. (1) suggests that each agent will at least have a minimal amount of capital. Nevertheless, there exists a distinct "accumulation barrier" in the model: a sufficiently low $h$ for a given agent ensures that the agent and the agent's offspring do not accumulate additional assets, which implies income stagnation. For an agent possessing a sufficiently high $h$, educational bequests will be large enough to ensure asset accumulation across generations. This implies income growth across generations, at least until the steady state level is reached. Given the above sketch of the model, we are now in a position to discuss the emergence of the Kuznets curve in the AR model.

Consider an autocracy where the rich are in control of the political system. AR assume that taxes and transfers cannot be agent specific, which implies that no taxation and transfer system will exist, as it would not be in the elite's best interest to support such a system. They also assume that rich agents have asset wealth above the accumulation barrier, but the poor agents do not. This implies that inequality-as measured by $y_{r, t} / y_{p, t}=h_{r, t} / h_{p, t}$-is increasing in $t$. The poor will eventually threaten to revolt if the payoff they get after a revolution exceeds their payoff under elite rule. This will happen when

$$
\frac{h_{r, t}}{h_{p, t}}>\frac{\lambda(1-\mu)}{\mu(1-\lambda)}
$$

where $\mu$ is the fraction of the capital stock that remains after the revolution. ${ }^{14}$ When Eq. (2) holds, the rich will choose to transfer political power to the poor in order to escape the threat of a revolution, setting up a democratic regime. From this period onwards the poor will vote for redistributive taxation, which permits their accumulation of capital and a reduction in inequality.

The EBM thus provides a straightforward account of the Kuznets hypothesis. That is, under autocracy inequality will be increasing, but after the transition to democracy redistributive taxation will promote decreasing inequality. Several questions, however, remain beyond the scope of the EBM. How does the emergence and evolution of the Kuznets curve depend on other institutions (e.g. interclass marriage, social mobility, and inheritance)? To what extent does this depend on heterogeneity among the agents, especially with respect to wealth? The model's agnostic position on these mechanisms limits both its scope and explanatory power. Is the model a surrogate or a substitute of reality? To what extent does it resemble real-world economies and is the mechanism outlined really the driver of the Kuznets-like pattern? ${ }^{15}$

\footnotetext{
14 All assets are shared equally among the poor agents if a revolution occurs. See Eq. (5) and associated text in AR for further discussion of this "revolution constraint."

15 The fact that the EBM is a static model makes it difficult to assess its predictions empirically since it does not say anything on the time horizon of the inequality dynamics. We discuss this aspect in more detail in Sect. 4.2. But we point out here that this illustrates the claim of Sect. 3 that ABMs are usually easier to validate than EBMs once data is available.
} 


\subsection{The Agent-Based Version}

We can remedy these shortcomings to some extent by transforming AR's EBM into an ABM. As opposed to using a continuum of agents, we begin by creating a population of $N=N_{r, t}+N_{p, t}$ discrete agents where $N_{i, t}$ denotes the number of agents of type $i$ at time $t$. We can then endow each agent with an asset $h_{i, t} \geq 1$ and give agents the same behavioral rules used in the EBM (e.g. asexual reproduction). The next step is to rewrite select conditions to reflect the discrete nature of the agents. For example, it is straightforward to show that Eq. (2) becomes

$$
\frac{h_{r, t}}{h_{p, t}}>\frac{N_{p}(1-\mu)}{\mu N_{r}}
$$

in the ABM. With these changes made (and the appropriate parameterization), our ABM can replicate the results of AR's EBM (see Sect. 4.3).

Our primary goal, however, is to extend the EBM. We do so in two respects: we introduce heterogeneous asset endowments for the agents and explicit mating institutions. To introduce heterogeneity in asset endowments, let $p_{j}=j / N$ where $j=1, \ldots, N$ indexes the agents and set the cumulative asset share of agents that own no more assets than agent $j$ to

$$
F\left(p_{j}\right)=1-\left(1-p_{j}\right)^{\delta}
$$

Equation (4) is the Lorenz curve associated with a Pareto distribution and $0<\delta \leq 1$ is the shape parameter (Eswaran and Kotwal 1986). At initialization, the asset endowment of agent $j$ is then given by

$$
h_{j}=H\left[F\left(p_{j}\right)-F\left(p_{j-1}\right)\right]
$$

where $H$ is the total quantity of assets available in the economy. By altering the parameter $\delta$, one can then examine how different asset distributions affect the evolution and emergence of the Kuznets curve.

Another particularly stringent assumption associated with the EBM is asexual reproduction. Recent empirical evidence suggests that positive assortative mating-the phenomenon that people reproduce with others who have similar characteristicsis an important driver of household inequality (Hou and Myles 2008; Torche 2010; Greenwood et al. 2014). Through our ABM we can naturally introduce more realistic mating mechanisms, including inter-class marriage. Let poor agents be those with a value of $h$ lower than the "accumulation barrier" and let rich agents be those with $h$ above the accumulation barrier. Further, let $\alpha$ be a parameter that controls the assortativity of mating.

For poor agents, the following expression provides the proportion of poor agents that mate with a poor agent in any given period:

$$
\alpha+\frac{N_{p}}{N}(1-\alpha)
$$


When $\alpha=0$ it is evident that random mating results, and when $\alpha=1$ it is evident that perfectly (positive) assortative mating results and the original setting can be reproduced. An analogous expression can be written for rich agents. These expressions can serve as the basis of a simple algorithm for "marrying" agents in each period. Moreover, one can use the parameter $\alpha$ and alternative inheritance institutions to examine how inter-class marriage affects wealth dynamics and thus the Kuznets curve.

\subsection{Results}

To illustrate that the model extensions serve to provide a richer account of observed historical experiences, Fig. 2 presents some selected results from the ABM using the parameterization discussed in Alvarez-Pereira et al. (2015). Results are the average of 20 simulation runs. The figure shows the time series of income inequality and poverty for different levels of mating assortativity (i.e., different levels of $\alpha$ ). Each unit of time corresponds to a generation. The height of each line captures income inequality, which is measured by the Gini coefficient, and the thickness of each line captures poverty, which is measured by the number of poor agents.

In Fig. 2 it is evident that there is a monotonic relationship between assortativity in mating and the onset of the Kuznets curve. In particular, as mating becomes more positively assortative, the onset of the Kuznets curve is delayed. Intuitively, the payoff of revolution to a given poor agent depends on the size of the post-revolution capital stock and the number of agents in each class (see Eq. 3). When mating is less assortative, the offspring of poor agents have an increased likelihood of escaping poverty

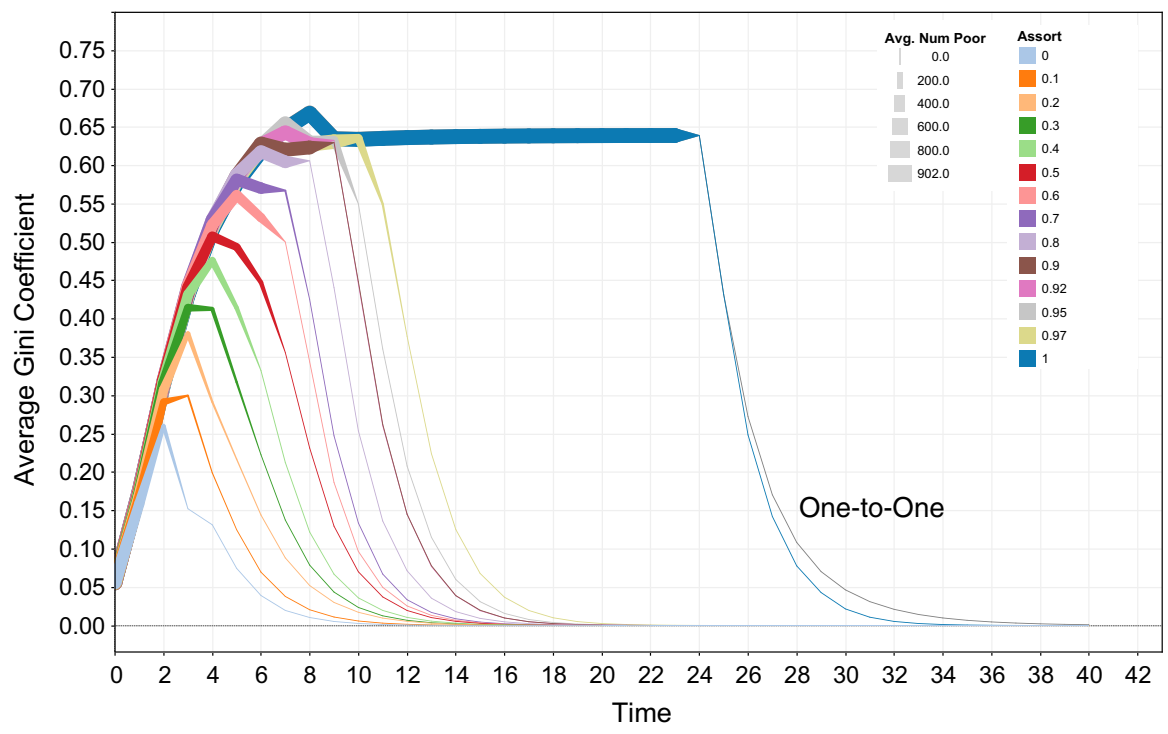

Fig. 2 Inequality dynamics of the agent-based model. $\alpha=0$ corresponds to random, $\alpha=1$ to perfectly assortative mating. One-to-one mating means that each agent "generates" her own child and passes on her savings directly 
and accumulating capital. By increasing total wealth, the hastened accumulation of capital coupled with a reduction in poverty makes the revolution optimal for the poor proportion of the population earlier in time. Conversely, when mating is more assortative, it is difficult for poor agents to escape poverty or accumulate capital, which tends to postpone the threat of revolution.

Acemoglu and Robinson (2000) examine the onset of the Kuznets curve for four European countries: Britain, France, Germany, and Sweden. They argued that the onset of the Kuznets curve occurred in the mid-to-late nineteenth century for Britain and France, but in the early-to-mid twentieth century for Germany and Sweden. Smits et al. (1998) provide estimates regarding the assortativity of mating in each of these countries. ${ }^{16}$ Most interestingly, the authors find that Britain is the least assortative whereas Germany witnesses the highest assortativity in mating. This evidence is broadly consistent with the results of our ABM, which suggest that countries with low assortativity should witness an earlier onset of the Kuznets curve. While this discussion of the empirical evidence is only suggestive, it does highlight the potential for the combination of EBMs and ABMs to provide new insights regarding real-world phenomena.

\subsection{Comparing the Equation-Based and Agent-Based Model}

Our application illustrates the benefits of extending EBMs using agent-based techniques. It exemplifies the complementary nature of EBMs and ABMs, also highlighting some practical difficulties. Here we compare and contrast the above-discussed models along five dimensions, ranging from the epistemological to the more practical.

First, using agent-based techniques, we were able to model the income distribution explicitly and calibrate it to empirical data. This increases the meaningfulness of the model through two channels. On one hand, the complexity reduction function of the model can be assessed more concretely. For example, does the model world share the same initial income distribution as the real world? On the other hand, by varying the income distribution, the model can be explored in a dimension that is not possible in the EBM. That is, we are able to understand how increasing inequality affects the shape and timing of the onset of the Kuznets curve. Understanding this dimension of the model is clearly relevant for understanding inequality dynamics in the real world.

Second, the ABM allows us to capture interaction and to relax the assumption of asexual reproduction. In particular, we are able to explore the effect of social mobility and alternative marriage institutions. The resulting increase of epistemic content is again twofold. First, sharing important similarities with reality strengthens the representative role of the model. This is particularly important given the increasing consensus in the empirical literature on the importance of marriage institutions for inequality dynamics. Second, we are able to explore the model in yet another

\footnotetext{
16 More specifically, the authors present estimates of "educational homogamy" for 65 countries using data from the 1970s and 1980s. Given that the asset in AR's model can be conceptualized as human capital, we believe that educational homogamy estimates are particularly relevant. Ideally, we would have estimates of mating assortativity for each country before the onset of the Kuznets curve, but such estimates do not appear to exist.
} 
dimension. In particular, we are able to examine how social mobility itself affects the evolution and emergence of the Kuznets curve. This extension may thus serve to provide a richer account of observed historical experiences.

Third, taking these extensions together, the model informs empirical testing of the Kuznets curve. The ABM predicts that the time horizon of the Kuznets curve will vary with differences in initial distributions of wealth, differing degrees of social mobility, and alternative inheritance institutions. Our consideration of Britain and Germany in Sect. 4.3 fits well into these new model predictions and suggests that the impact of initial inequality and assortativity on the dynamics of the Kuznets hypothesis must be taken into account should the hypothesis be tested empirically. In this way, our results suggest that existing studies may suffer from issues of omitted variable bias, which may help explain the weak evidence on Kuznets' hypothesis (Alvarez-Pereira et al. 2015). The ABM thus points toward more precise empirical tests of the resemblance aspect of the model.

Fourth, the modular structure of the ABM allows the precise isolation of the effects of different initial wealth distributions and different degrees of social mobility. That is, the ABM nests the EBM, and the extensions can be activated or deactivated as the researcher finds appropriate. This ability to isolate the effects of additional mechanisms is crucial, as adding a new mechanism is useless if the consequences cannot be isolated precisely. In such a case, they blur the former isolations and decrease the explanatory power of the model (Knoeri et al. 2014).

Finally, the development of an ABM based on an existing EBM presents a number of practical difficulties. First, parameterizing the ABM is not always straightforward. One typically needs to identify and gather the relevant data, and sensitivity analysis becomes an important part of model development. Second, the mapping of a mechanism of an $\mathrm{EBM}$ to a mechanism in an ABM is not always one-to-one. This is particularly true of incomputable mechanisms such as rational expectations (Velupillai 2009). ${ }^{17}$ Third, if a new aspect is introduced (e.g. explicit wealth distribution), questions about the exact implementation must often be answered in an ad hoc manner. Even if different choices likely lead to the same result, this presumption must be explored. This is often a tedious task, and conclusive proofs about the equivalence of two mechanisms are not available in a simulation context.

In our case, the ABM and EBM were complementary and our insights would not have been possible without both. The EBM provided concise characterization of the system-level signals within the model, giving us a model with which to dock the ABM. The ABM was able to implement mechanisms that would be infeasible with the EBM, thus extending the scope of the underlying theoretical model.

\footnotetext{
17 In such a case, the effect of rational expectations has to be approximated by similar mechanisms such as adaptive expectations. This can be particularly problematic if direct interdependence among the agents plays an important role. Fortunately, this was not a problem for our illustrative example. But if required one may use one of the three concepts proposed by Axtell et al. (1996) to establish the equivalence between ABMs and EBMs with rational expectations.
} 


\section{Discussion}

The preceding section described the process of building an ABM from an underlying EBM and illustrated what is meant by the increased explanatory power associated with the ABM. It also demonstrated the aspects of the MISS introduced previously. In this section, we offer discussion of our approach in light of the illustrative example. We first argue that the presentation of an ABM generally benefits from an explicit clarification of its epistemological base (i.e., by clarifying how it generates knowledge). The MISS framework is a simple and powerful device to do this, as it provides the theory and language to substantiate statements about the explanatory power of models.

With respect to our illustration of the Kuznets model, the explicit use of an epistemological framework in our argument has several advantages from both a theoretical and practical perspective. If one wishes to make inferences from a model to the real world, a certain epistemological foundation is required. If this foundation remains implicit, the model is less comparable to alternatives and readers are forced to speculate about where the alleged increased explanatory power of the model comes from. The MISS framework is very clear in this respect: the epistemic content of a model derives from its adequacy to represent and resemble the real world. Representation is a necessary condition for resemblance, and only if the model resembles the real world is it said to have epistemic content.

The distinction between the representative aspect and the resemblance aspect of a model is particularly useful for ABMs, as justifying an ABM usually involves statement about both aspects. The overall message can be made much clearer by taking this distinction seriously. Just as a common form of describing the functioning of simulation models - such as the ODD protocol of Grimm et al. (2010) or Baumgärtner et al. (2008) — facilitates the communication of ABMs, a common form for describing their underlying epistemology would facilitate the comparison of different models and thus foster the scientific discourse more generally. This is particularly the case for the discours within the ABM community where many different epistemologies are currently used, but rarely communicated explicitly.

Which particular epistemological approach is used for a given model often depends on its audience and purpose (Mäki 2009b). Some models are used to produce predictions while other models are used to illustrate certain mechanisms in the real world. Different types of models not only require very different validation techniques, but they also require different epistemological justifications and therefore cannot be easily compared to each other.

One advantage of the MISS framework is to provide an adequate meta-language to consider the concepts of "audience" and "purpose" when discussing a model (Mäki 2009a). In particular, the MISS diagram in Fig. 1 provides an intuitive visual means to support this discussion. Many analytical models are well suited for providing predictions about the real world and thus to infer states from $S_{2}$ to $R_{2}$. In such cases, priority is given to simple but precise transformation functions. Models built for explanatory purposes are used to infer mechanisms. Consequently, they give priority to more complex transformation functions that allow for a generative description of the model (Epstein 1999). While we do not argue for the superiority of one type of model over 
the other, we do believe that for reasons of comparability their purpose should be made explicit. The MISS provides a good framework to do so.

Contrary to what is common in the literature, the MISS framework suggests complementarity rather than competition between ABM and EBM. In our case, while the ABM has many advantages over the EBM, it is the original EBM that helps to discipline the parameterization of the ABM, thereby keeping the central message clear. In our approach, one is always forced to test whether the additional mechanisms truly contribute to the understanding of the system under investigation. New mechanisms are added step-by-step to allow for an adequate isolation of their consequences. That is, one has to balance the disaggregation and descriptive richness provided by ABM with the aggregation and rigor of the associated EBM.

\section{Concluding Remarks}

Several key messages arise from our approach. First, using an explicit epistemological framework for simulation models allows researchers to communicate the usefulness of their model in a more transparent and straightforward way. The MISS framework is particularly well suited for the ABM case as it provides the necessary meta-language to discuss why a particular model is useful for a particular purpose, how it produces knowledge about the SUI, and how it relates to alternative models. If no explicit epistemological framework is used, all related considerations remain implicit and model assessment and comparison become difficult. Just as the ODD protocol is an excellent framework to describe the technical functioning of an ABM, the MISS framework is well suited to explain the epistemological content of an ABM

Second, building agent-based versions of EBMs is a fruitful endeavor. An ABM can generate a substantial amount of additional knowledge about the target system. Additionally, it can be useful for identifying the implicit assumptions underlying the corresponding EBM and examining its adequacy as a surrogate system for the problem at hand. Third, ABMs are not necessarily substitutes for EBMs. In fact, the intermediate step of building an EBM is a useful exercise, as it helps to limit the disadvantages associated with ABMs. In particular, it permits easier communication of the model to the target audience and disciplines the modeler to focus on the central mechanisms. Finally, EBMs are often amendable to proofs and thus easier to verify. Building an agent-based version of a rigorously verified EBM involves much less speculation and uncertainty than building an ABM from scratch. As such, our approach is also in line with the demand for a methodological pluralism in the social sciences, as one perspective alone is hardly enough to tackle the complex problems of our time (see also Ostrom and Basurto 2011 or Gräbner 2017a).

Our sequential strategy illustrates that a rigorous demarcation between ABM and EBM is often not very useful. The most pragmatic and useful differentiation goes along epistemological properties: speaking in the terms of MISS, ABMs tend to focus more on an adequate representation of reality rather than on the verification of the model as such. Underlying this are different "weights" for the representation aspect of economic models and the question of whether models should focus more on resembling states or mechanisms. 
While our approach holds promise, it is also associated with some practical difficulties. It is not always straightforward or possible to translate an EBM into an ABM. Furthermore, collecting data for the system in question is not a trivial task and the parameterization of an ABM can often seem ad hoc. Overcoming these challenges is a difficult, yet important task (see Fagiolo et al. 2007 for constructive proposals). Our attempt at building an ABM from Acemoglu and Robinson's (2002) EBM illustrates the usefulness of the approach and we hope that it inspires further research.

Acknowledgements The authors would like to thank colleagues at the Santa Fe Institute's 2014 Complex Systems Summer School for helpful discussion and insights that led to the ideas presented in this paper. CSEB would like to acknowledge the Engineering and Physical Sciences Research Council for funding under Grant EP/K022288/1. CG would like to acknowledge funding by the University of Bremen through the research impulse program and by the Austrian National Bank (OeNB) under Grant Number 17383. BAF would like to acknowledge grant from the National Council of Research under Grant 306954/2016-8. The authors gratefully acknowledge open access funding provided by the Johannes Kepler University Linz.

Open Access This article is distributed under the terms of the Creative Commons Attribution 4.0 International License (http://creativecommons.org/licenses/by/4.0/), which permits unrestricted use, distribution, and reproduction in any medium, provided you give appropriate credit to the original author(s) and the source, provide a link to the Creative Commons license, and indicate if changes were made.

\section{References}

Acemoglu, D., \& Robinson, J. A. (2000). Why did the west extend the franchise? Democracy, inequality, and growth in historical perspective. The Quarterly Journal of Economics, 115(4), 1167-1199.

Acemoglu, D., \& Robinson, J. A. (2002). The political economy of the Kuznets curve. Review of Development Economics, 6(2), 183-203.

Albin, P., \& Foley, D. K. (1992). Decentralized, dispersed exchange without an auctioneer: A simulation study. Journal of Economic Behavior \& Organization, 18(1), 27-51.

Alvarez-Pereira, B., Bale, C.S.E., Alves Furtado, B., Gentile, J.E., Gräbner, C., Henderson, H., \& Lipari, F. (2015). Social institutions and economic inequality: Modeling the onset of the Kuznets curve. IPEA Discussion Paper No. 204.

Andersen, P. B., Emmeche, C., Finnemann, N. O., \& Christiansen, P. V. (2000). Downward causation. Aarhus: Aarhus University Press.

Arrow, K. J., \& Debreu, G. (1954). Existence of an equilibrium for a competitive economy. Econometrica, 22(3), 265-290.

Arthur, W. B. (1989). Competing technologies, increasing returns, and lock-in by historical events. The Economic Journal, 99(394), 116-131.

Axtell, R. (2005). The complexity of exchange. The Economic Journal, 115(504), F193-F210.

Axtell, R., Axelrod, R., Epstein, J., \& Cohen, M. (1996). Aligning simulation models: A case study and results. Computational \& Mathematical Organization Theory, 1(2), 123-141.

Baumgärtner, S., Becker, C., Frank, K., Müller, B., \& Quaas, M. (2008). Relating the philosophy and practice of ecological economics: The role of concepts, models, and case studies in inter- and transdisciplinary sustainability research. Ecological Economics, 67, 384-393.

Cecconi, F., Campenni, M., Andrighetto, G., \& Conte, R. (2010). What do agent-based and equation-based modelling tell us about social conventions: The clash between ABM and EBM in a congestion game framework. Journal of Artificial Societies and Social Simulation, 13(1), 6.

Durlauf, S. (2012). Complexity, economics, and public policy. Politics, Philosophy \& Economics, 11(1), $45-75$.

Epstein, J. (1999). Agent-based computational models and generative social science. Complexity, 4(5), 41-60.

Epstein, J. (2006). Remarks on the foundations of agent-based generative social science. In L. Tesfatsion \& K. Judd (Eds.), Handbook of computational economics (pp. 1586-1604). Amsterdam: Elsevier/NorthHolland. 
Eswaran, M., \& Kotwal, A. (1986). Access to capital and agrarian production organisation. The Economic Journal, 96(382), 482-498.

Fagiolo, G., Moneta, A., \& Windrum, P. (2007). A critical guide to empirical validation of agent-based models in economics: Methodologies, procedures, and open problems. Computational Economics, 30, 195-226.

Friedman, M. (1953). The methodology of positive economics. In M. Friedman (Ed.), Essays in Positive Economics (pp. 3-16, 30-43). Chicago, NJ, USA: University of Chicago Press.

Furtado, B. A., \& Sakowski, P. A. M. (2014). Complexity: A review of the classics. Journal on Policy and Complex Systems, 1(2), 4-18.

Gilboa, I., Postlewaite, A., Samuelson, L., \& Schmeidler, D. (2014). Economic models as analogies. The Economic Journal, 124(578), F513-F533.

Gintis, H. (2007). The dynamics of general equilibrium. The Economic Journal, 117, 1280-1309.

Gräbner, C. (2017a). Dealing adequately with the political element in formal modelling. In H. Hanappi, S. Katsikides, \& M. Scholz-Wäckerle (Eds.), Theory and method of evolutionary political economy (Vol. 2, pp. 236-254). London and New York, NY: Routledge.

Gräbner, C. (2017b). The Complementary Relationship Between Institutional and Complexity Economics: The Example of Deep Mechanismic Explanations. Journal of Economic Issues, 51(2), 392-400.

Gräbner, C., \& Kapeller, J. (2015). New perspectives on institutionalist pattern modeling: Systemism, complexity, and agent-based modeling. Journal of Economic Issues, 49(2), 433-440.

Greenwood, J., Guner, N., Kocharkov, G., \& Santos, C. (2014). Marry your like: Assortative mating and income inequality. American Economic Review, 104(5), 348-353.

Grimm, V., Berger, U., DeAngelis, D. L., Polhill, J. G., Giske, J., \& Railsback, S. F. (2010). The ODD protocol: A review and first update. Ecological Modelling, 221(23), 2760-2768.

Halloran, M. E., Longini, I. M, Jr., Nizam, A., \& Yang, Y. (2002). Containing Bioterrorist Smallpox. Science, 298(5597), 1428-1432.

Henderson, H., \& Isaac, A. G. (2017). Modern value chains and the organization of Agrarian production. American Journal of Agricultural Economics, 99(2), 379-400.

Hodgson, G. M., \& Knudsen, T. (2004). The complex evolution of a simple traffic convention: The functions and implications of habit. Journal of Economic Behavior \& Organization, 54(1), 19-47.

Hou, F., \& Myles, J. (2008). The changing role of education in the marriage market: Assortative marriage in Canada and the United States since the 1970s. Canadian Journal of Sociology, 33(2), 337-366.

Johnson, J., Ormerod, P., Rosewell, B., Nowak, A., \& Zhang, Y. (2017). Non-equilibrium social science and policy understanding complex systems. New York: Springer.

Keeling, J. (1999). The effects of local spatial structure on epidemiological invasions. Proceedings of the Royal Society B: Biological Sciences, 266(1421), 859-867.

Knoeri, C., Nikolic, I., Althaus, H. J., \& Binder, C. R. (2014). Enhancing recycling of construction materials: An agent based model with empirically based decision parameters. Journal of Artificial Societies and Social Simulation, 17(3), 10.

Kuznets, S. (1955). Economic growth and income inequality. American Economic Review, 45(1), 1-27.

Lafuerza, L. F., Dyson, L., Edmonds, B., \& McKane, A. J. (2016). Staged models for interdisciplinary research. PLOS ONE, 11(6), e0157261.

Mäki, U. (2009a). MISSing the world. Models as isolations and credible surrogate systems. Erkenntnis, $70(1), 29-43$.

Mäki, U. (2009b). Models and truth: The functional decomposition approach. In M. Suárez, M. Rédei, \& M. Dorato (Eds.), EPSA Epistemology and methodology of science: Launch of the European Philosophy of Science Association (pp. 177-187). Dordrecht: Springer.

Musgrave, A. (1981). 'Unreal assumptions' in economic theory: The F-twist untwisted. Kyklos, 34(3), 377-387.

Oremland, M., \& Laubenbacher, R. (2014). Using difference equations to find optimal tax structures on the SugarScape. Journal of Economic Interaction and Coordination, 9(2), 233-253.

Ostrom, E., \& Basurto, X. (2011). Crafting analytical tools to study institutional change. Journal of Institutional Economics, 7(3), 317-343.

Picard, N., \& Franc, A. (2001). Aggregation of an individual-based space-dependent model of forest dynamics into distribution-based and space-independent models. Ecological Modeling, 145, 69-84.

Pyka, A., \& Fagiolo, G. (2007). Agent-based modelling: A methodology for neo-Schumpeterian economics. In H. Hanusch \& A. Pyka (Eds.), Elgar Companion to Neo-Schumpeterian Economics (pp. 467-487). Cheltenham, UK and Northampton, MA, USA: Edward Elgar. 
Rahmandad, H., \& Sterman, J. (2008). Heterogeneity and network structure in the dynamics of diffusion: Comparing agent-based and differential equation models. Management Science, 54(5), 998-1014.

Rust, J. (1998). Dealing with the complexity of economic calculations. In S. Durlauf \& J. Traub (Eds.), Limits to Knowledge in Economics. Boston MA: Addison-Wesley.

Smits, J., Ultee, W., \& Lammers, J. (1998). Educational homogamy in 65 countries: An explanation of differences in openness using country-level explanatory variables. American Sociological Review, 63(2), 264-285.

Sudgen, R. (2000). Credible Worlds: the status of theoretical models in economics. Journal of Economic Methodology, 7(1), 1-31.

Sukumar, S., \& Nutaro, J. (2012). Agent-based vs. equation-based epidemiological models a model selection case study. ASE/IEEE international conference on BioMedical computing (BioMedCom) (pp. 74-79).

Torche, F. (2010). Educational assortative mating and economic inequality: A comparative analysis of three Latin American Countries. Demography, 47(2), 481-502.

Van Dyke Parunak, H., Savit, R., \& Riolo, R. (1998). Agent-based modeling vs. equation-based modeling: A case study and users' guide. In J.S. Sichman, R. Conte \& N. Gilbert (Eds.), Multi-agent systems and agent-based simulation (pp. 10-25). Berlin: Springer.

Velupillai, K. V. (2009). Uncomputability and undecidability in economic theory. Applied Mathematics and Computation, 215, 1404-1416. 